\title{
A Degree-Day Model for the Latent Period of Stagonospora nodorum Blotch in Winter Wheat
}

A. D. Zearfoss, Department of Plant Pathology, North Carolina State University, Raleigh, NC 27695; C. Cowger, USDA-ARS, Department of Plant Pathology, North Carolina State University, Raleigh, NC 27695; and P. S. Ojiambo, Department of Plant Pathology, North Carolina State University, Raleigh, NC 27695

\begin{abstract}
Zearfoss, A. D., Cowger, C., and Ojiambo, P. S. 2011. A degree-day model for the latent period of Stagonospora nodorum blotch in winter wheat. Plant Dis. 95:561-567.

Stagonospora nodorum blotch (SNB), which is caused by Stagonospora nodorum, occurs frequently in the southeastern United States, and severe epidemics can lead to substantial yield losses. To develop a model for the progress of SNB based on the effects of temperature on the latent period of the pathogen, batches of two winter wheat cultivars, AGS 2000 and USG 3209, were inoculated with S. nodorum at weekly intervals for 16 weeks. After $72 \mathrm{~h}$ of incubation, inoculated plants were exposed to outdoor conditions where temperatures ranged from -6.6 to $35.8^{\circ} \mathrm{C}$, with a mean batch temperature ranging from 9.7 to $24.7^{\circ} \mathrm{C}$. Latent period, expressed as time from inoculation until the first visible lesions with pycnidia, ranged from 13 to 34 days. The relationship between the inverse of the latent period and mean

thermal time required for the completion of the latent period was 384.6 degree-days. A shifted cumulative gamma distribution model with a base temperature of $0.5^{\circ} \mathrm{C}$ significantly $(P<0.0001)$ described the relationship between increasing number of lesions with pycnidia and accumulated thermal time. When latent period was defined as time to $50 \%$ of the maximum number of lesions with pycnidia $(L 50)$, the model estimated $L 50$ as 336 and 326 degree-days above $0.5^{\circ} \mathrm{C}$ for AGS 2000 and USG 3209, respectively. The relationship between $1 / L 50$ and mean temperature was also best described using a linear model $\left(r^{2}=\right.$ $0.93, P<0.001)$. This study provides data that link disease progress with wheat growth, which facilitates accurate identification of thresholds for timing of fungicide applications.
\end{abstract} temperature was best described by a linear model, and the estimated
Stagonospora nodorum blotch (SNB), which is caused by Stagonospora nodorum (teleomorph Phaeosphaeria nodorum), is an important disease of wheat leaves, stems, and glumes $(10,36)$. The disease is widely distributed in major wheat-growing regions of the world and is especially significant in areas with warm and humid conditions (36), such as the southeastern United States. Changes in cultural practices, such as planting at higher densities with shorter rotations and the increased use of fertilizers, have increased the intensity of the disease $(18,37)$. The disease affects both grain quality and yield. The milling quality of grain is reduced by infection of the developing seed, which results in shriveling and discoloration of the grain and reduced flour yield $(5,6,24)$. The disease can also result in reduced grain yield and lower test weights $(5,6,26)$. This is due to reduced photosynthetic area of the upper leaves that results in less carbohydrates for grain fill (11). Grain yield losses of up to $31 \%$ have been reported in areas where environmental conditions favor disease epidemics $(5,10,25)$.

The primary source of initial infection of wheat by $S$. nodorum is thought to be either ascospores $(2,33)$ or infected seed $(3,29)$. Disease development during the season is primarily due to pycnidiospores that are spread by rain-splash from the lower parts of the canopy to upper leaves (9). Colonization of infected host tissues leads to formation of lesions that often contain fruiting bodies known as pycnidia. The effects of environmental factors such as temperature on the pathogen's latent period and life cycle are important for establishing models of disease development that can be used to improve disease control strategies (27). The term "latent

Corresponding author: P. S. Ojiambo, E-mail: peter_ojiambo@ncsu.edu

Accepted for publication 4 January 2011.

doi:10.1094/PDIS-09-10-0651

This article is in the public domain and not copyrightable. It may be freely reprinted with customary crediting of the source. The American Phytopathological Society, 2011. period" denotes the time between infection and the first appearance of fungal sporulating structures (31), and is an important parameter that affects the rate at which epidemics develop in crops. The latent period of $S$. nodorum is relatively long when compared to that of other foliar pathogens of wheat. However, reported values for the latent period vary greatly: 6 to 49 days (31), 11 to 16 days (34), 9 to 37 days (15), 21 to 34 days (20), and 24 days (19). Time to development of disease symptoms is strongly influenced by environmental conditions such as moisture and temperature, with an estimated optimum temperature in the range of 20 to $25^{\circ} \mathrm{C}$ (36). For example, little or no development of SNB has been observed when the minimum temperature is $\leq 7^{\circ} \mathrm{C}$ regardless of the high relative humidity provided after inoculation (13).

The growth and development of plant pathogens and their hosts generally respond strongly to the temperature of their environment. In this regard, thermal time is useful in the assessment and modeling of growth and development of many plant pathogens (23). In an effort to describe the degree to which host growth affects the expression of foliar diseases on crops, Beresford and Royle (4) introduced the concept of the "pathochron", which is the ratio of thermal time (degree-days above a $0^{\circ} \mathrm{C}$ base temperature) for the pathogen's latent period to the thermal time for the emergence of successive host leaves (phyllochron). Simply put, a pathochron describes the number of leaves emerging within a latent period of the pathogen. Sporulation of the pathogen cannot occur until a leaf is at least one latent period old, and during a single latent period, new leaves can emerge. Thus, the latent period of the pathogen interacts with host growth to influence the spatial development of a disease within a crop. Using these concepts, rates of stem extension and leaf emergence were indentified as factors that could affect the risk of Septoria leaf blotch (caused by Septoria tritici) on winter wheat crops (22). For example, emerging upper canopy leaves can grow away from infected lower leaves during stem elongation, and splash dispersal is needed for spores to reach leaves in the upper canopy. Based on the latent period data from Shaw (30) and phyllochron data developed by Kirby (17), it was demonstrated that the number of leaves emerging within a latent period of Septo- 
ria leaf blotch increased with increasing temperature (22). This indicated that at higher temperatures, emerging upper leaves of a wheat crop are more likely to grow away from infected lower leaves more quickly, thereby reducing the likelihood of the deposition of $S$. tritici spores on upper canopy leaves.

Previous studies on the effect of temperature on the latent period of $S$. nodorum on wheat were conducted under controlled conditions in either a greenhouse or a growth chamber $(19,31,34)$. Thus, results from these studies may not reflect the interaction between the latent period of $S$. nodorum and temperature experienced under field conditions. To be useful in predicting conditions when economic damage may occur, $S$. nodorum latent period data should be acquired under a range of temperature regimes in the field. Since leaf emergence is constant during the development of wheat, the latent period could be used to adjust for crop growth to ensure that leaves of appropriate age are monitored for SNB when making decisions on timing of fungicide applications. Thus, the objective of this study was to determine the effect of temperature on the latent period of $S$. nodorum in winter wheat under field conditions. The working hypothesis was that the latent period measured on a thermal time scale could be defined for $S$. nodorum, and when coupled with host growth, the latent period on a thermal time scale would be useful in monitoring the disease and will facilitate decision making for the management of Stagonospora nodorum blotch.

\section{Materials and Methods}

Test genotypes and field site. Two cultivars of soft red winter wheat (Triticum aestivum), AGS 2000 and USG 3209, were used in this study. These cultivars are widely grown in the southeastern United States and have been utilized as parents in developing cultivars for the region. These cultivars differ in their response to infection by $S$. nodorum, with AGS 2000 being relatively more susceptible to SNB than USG 3209 (7). Batches of seedlings of each cultivar were inoculated (see below) with $S$. nodorum at weekly intervals from February to June 2009. Following a period of incubation, inoculated seedlings were exposed to outdoor conditions at the USDA-ARS Reedy Creek Road Field Laboratory in Raleigh, NC, which was at least $10 \mathrm{~km}$ from fields with wheat or wheat stubble.

Preparation of inoculum and plants. S. nodorum isolate NC-71 was used for inoculum production. NC-7-1 was isolated from a lesion on wheat debris collected in 2008 from a field trial in Rowan County, NC, and maintained as a conidial suspension in $80 \%$ glycerol at $-80^{\circ} \mathrm{C}$. Fresh inoculum was obtained weekly by subculturing $0.2-\mathrm{ml}$ aliquots of the stock onto petri plates containing V8 medium (200 $\mathrm{ml}$ of $\mathrm{V} 8$ juice, $3 \mathrm{~g}$ of $\mathrm{CaCO}_{3}, 15 \mathrm{~g}$ of agar, and 800 $\mathrm{ml}$ of sterile distilled water). The cultures for inoculum production were grown in a growth chamber at $22^{\circ} \mathrm{C}$ under continuous black light (near-UV, $360 \mathrm{~nm}$ ) to promote sporulation. Inoculum was obtained by flooding the surface of a 7-day-old culture with sterile distilled water and then scraping the agar surface with a sterilized glass rod to release spores. The final spore suspension was adjusted to $1 \times 10^{6}$ spores/ml by dilution, using a hemacytometer. Prior to inoculation (described below), a germination test was conducted using cavity slides as described by Lovell et al. (21) to determine the viability of pycnidiospores. The germination frequency of pycnidiospores was $>95 \%$ for the spore suspensions used to inoculate all batches of wheat seedlings.

Seedlings of AGS 2000 and USG 3209 were grown in 9-cm pots in a controlled-environment chamber at $21^{\circ} \mathrm{C}$ until the emergence of the second leaf and then moved to a greenhouse where temperatures were maintained at $24^{\circ} \mathrm{C}$. A total of eight pots per cultivar were used for each weekly batch of seedlings. The greenhouse conditions allowed the plants to avoid natural infection due to $S$. nodorum prior to inoculation. When control of powdery mildew was necessary, soil in each pot was drenched with $50 \mathrm{ml}$ of the fungicide ethirimol (15). Ethirimol is a narrow-spectrum fungicide that is effective against powdery mildew but has no activity against $S$. nodorum (12). After the first four leaves were fully emerged, pots were thinned to two plants per pot, and the second, third, and fourth leaves were marked to facilitate the identification of the leaves during assessment of plants for disease symptoms in the field.

Inoculation procedure. At approximately weekly intervals from February to June 2009, a batch of AGS 2000 and USG 3209 seedlings was inoculated with $S$. nodorum and exposed to ambient temperatures. A batch consisted of eight plants per cultivar (16 plants total), of which six plants of each cultivar were inoculated and two were used as noninoculated controls. Seedling plants were placed on a rotating turntable and sprayed with a spore suspension $\left(1 \times 10^{6}\right.$ spores $\left./ \mathrm{ml}\right)$ containing one drop of Tween 20 until runoff, using a handheld spray bottle. To ensure successful infection after inoculation, each pot was enclosed in a separate polyethylene bag with distilled water in the base to maintain high humidity and then placed in a growth chamber at $21^{\circ} \mathrm{C}$ with $12 \mathrm{~h}$ daylight. After $48 \mathrm{~h}$, the polyethylene bags were removed. The plants remained in the growth chamber $\left(12 \mathrm{~h}\right.$ daylight and $\left.21^{\circ} \mathrm{C}\right)$ for an additional $24 \mathrm{~h}$, after which they were exposed to outdoor conditions at the USDAARS research facility. Hourly records of temperatures at the experimental site were monitored using data loggers (WatchDog, Spectrum Technologies, Plainfield, IL) housed in a Gill radiation screen positioned about $100 \mathrm{~cm}$ above the pots.

Assessment of disease symptoms and definition of latent period. To assist in monitoring of symptoms, leaves 2 to 4 (from the base of the plant) were tagged prior to exposing plants to outdoor conditions. Disease symptoms were assessed as the number of lesions with one or more visible pycnidia of $S$. nodorum. Lesions were assessed for pycnidia with the aid of a jeweler's lens with $\times 4.5$ magnification, and each lesion with pycnidia and the leaf on which it was found were recorded. Disease assessment was conducted every 2 to 3 days from the time of inoculation until leaf senescence or until numbers of lesions with pycnidia remained constant in at least four successive assessments of disease symptoms.

Generally, latent period is taken to denote the time from infection to appearance of sporulating structures (31). However, Shaw (30) noted that this standard definition of the latent period has limited value if symptom expression is not synchronized across plants infected at the same time. This lack of synchronicity in symptom expression can be avoided if latent period is expressed as time to appearance of a population of lesions with sporulating structures. Here, we defined latent period in various ways to allow for comparisons with other studies and to generate estimates that could be useful for a range of conditions. Thus, the latent period for $S$. nodorum was defined as the time from inoculation to: (i) first lesions with visible pycnidia (LFVP), (ii) $5 \%$ of maximum lesions with symptoms (L5), (iii) $50 \%$ of maximum lesions with symptoms (L50), and (iv) maximum observed lesions with pycnidia (L100). In all cases, time was quantified in both days and accumulated temperature as described below. In addition, symptom duration, i.e., the time between first and last observed lesions with pycnidia, was calculated for AGS 2000 and USG 3209.

Data analysis. Latent period variables LFVP and $L 100$ were estimated from observed number of lesions with pycnidia collected over time, while $L 5$ and $L 50$ were estimated from a model (described below) used to describe the relationship between increasing number of lesions with pycnidia and accumulated thermal time. Following some preliminary analysis, the relationship between the daily rate of development during the latent period (1/LFVP) and mean temperature was examined separately for AGS 2000 and USG 3209 by linear regression analysis using the REG procedure of SAS version 9.2 (SAS Institute, Cary, NC). Homogeneity of slopes for AGS 2000 and USG 3209 was tested using the GLM procedure of SAS to determine if a common slope could be fitted to the reciprocal of LFVP and mean temperature across the two cultivars.

A method for solving systems of differential equations relating changes in measurable environmental variables such as temperature to development of an organism was proposed by Powers et al. (28). In this method, parameters are written as functions of environmental variables, and these functions involve the accumulation of "environment time", for example, accumulated degree-days (28). This method has also been used to develop a model to de- 
scribe the relationship between accumulated thermal time and development of $S$. tritici lesions on winter wheat (21). Here, we adopted a similar modeling approach to describe the increase in number of $S$. nodorum lesions with pycnidia with accumulation of thermal time (in degree-hours) above a base temperature (a temperature below which no development of lesions with pycnidia occurs). This model can be summarized as follows, and the details of the model are described in Powers et al. (28):

$$
N(t)=\left\{\begin{array}{ccc}
0, & T T(t)<p \\
N_{\max } & \int_{0}^{T T(t)-p} \frac{\alpha^{\beta}}{\Gamma(\beta)} u^{\beta-1} e^{-\alpha u} d u, & T T(t) \geq p
\end{array}\right\}
$$

where $T T(t)=\int_{0}^{t}(\operatorname{Temp}(u)-$ Tbase $) d u$ is the accumulated thermal time up to time $t$, in which $\operatorname{Temp}(u)=\left(T e m p_{\max }+T e m p_{\min }\right) / 2$ based on hourly data and the accumulation occurs only when $\operatorname{Temp}(u)$ is above the base temperature (Tbase). The model assumes that lesions with pycnidia will only develop after a latent period (expressed as thermal time, including the $72 \mathrm{~h}$ in the growth chamber has elapsed). The parameter $N_{\max }$ is the maximum number of lesions with pycnidia that develop from a given inoculation, while $p$ is the estimated thermal time from inoculation to the appearance of the first lesion with pycnidia (i.e., symptom lag). $\Gamma(\beta)$ is the gamma function (1), and $\alpha$ and $\beta$ are the shape and scale parameters, respectively, of the gamma distribution.

To facilitate fitting data to the model, data sets were summarized separately for AGS 2000 and USG 3209. These data sets were composed of increasing number of lesions with pycnidia over thermal time for each cultivar and batch of seedlings. After preliminary analysis to assess variability among pots within a batch of seedlings, data for number of lesions with pycnidia were pooled across pots, plants, and leaves to provide a mean summary of numbers of lesions with pycnidia for each batch of seedlings. The shifted gamma distribution model was fitted to these pooled data over all batches separately for each cultivar, using the NLIN procedure in SAS with the CDF function. The fitted model was then used to estimate $L 5$ and $L 50$ as multiplicative parameters of the distribution. For example, $L 50$ was estimated by replacing $N_{\max }$ with $0.5 \times$ $N_{\max }$ in the model. Goodness-of-fit of the model was evaluated based on magnitude of asymptotic confidence intervals of parameter estimates, asymptotic simple correlation between observed and predicted number of lesions with pycnidia, and plots of residuals versus predicted number of lesions with pycnidia.

\section{Results}

Temperatures and lesions with pycnidia. The effect of temperature on the number of lesions with pycnidia under field condi-

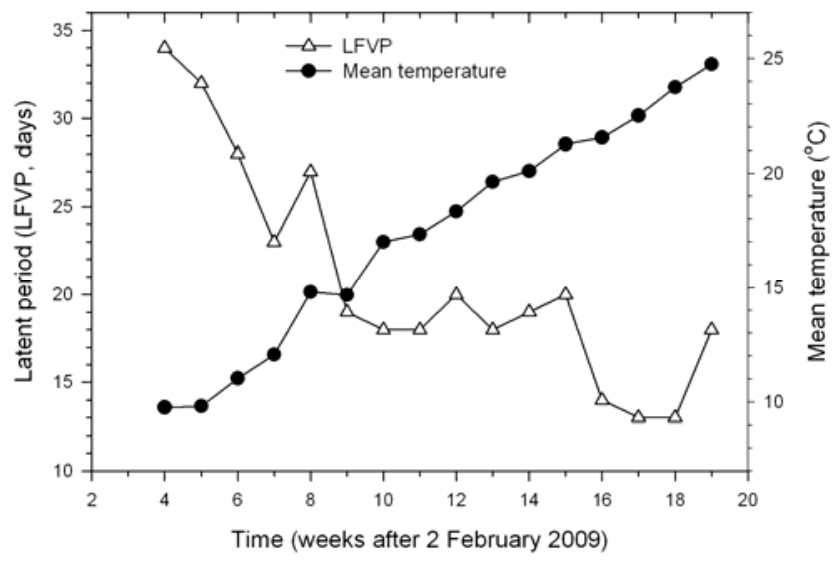

Fig. 1. Latent period (days) from inoculation of winter wheat with Stagonospora nodorum pycnidiospores to first lesions with pycnidia (LFVP) and corresponding mean air temperature for 16 batches of the wheat cultivar USG 3209 exposed to outdoor conditions from February to June 2009. Data points are plotted on dates when seedling batches were inoculated. tions was assessed based on a total of 16 batches of inoculated seedlings of wheat cultivars AGS 2000 and USG 3209. Batches of inoculated plants were exposed to field conditions from 2 February to 9 July 2009, during which temperatures ranged from -6.6 to $35.8^{\circ} \mathrm{C}$. The mean temperature from inoculation to development of lesions with pycnidia ranged from 9.8 to $23.7^{\circ} \mathrm{C}$. Generally, the mean temperatures to which batches of plants were exposed increased steadily during the experimental period, being lowest in February and highest in July (Fig. 1). Unlike the mean temperature, the latent period decreased over the study period, being longer in February and shorter in July (Fig. 1). In addition, there was considerable variation among batches in the maximum number of lesions with pycnidia per leaf. For example, the maximum number of lesions with pycnidia across all the batches ranged from 2.4 to 13.5 for USG 3209 and from 2.9 to 14.0 for AGS 2000, with a mean of $5.3( \pm 0.91)$ and $7.1( \pm 0.88)$, respectively. The maximum number of lesions with pycnidia and the mean temperature to which seedling batches were exposed were not significantly correlated for AGS $2000(P=0.11)$ and USG $3209(P=0.20)$.

Time to first visible lesions with pycnidia. Time from inoculation to the first lesions with visible pycnidia (LFVP) varied between 13 and 34 days for USG 3209. Generally, LFVP decreased with increasing temperature, with the curve taking the form of a hyperbolic function (Fig. 1). Similarly, LFVP varied between 8 and 32 days for AGS 2000, with LFVP decreasing with increasing temperature in a hyperbolic manner (data not shown). When expressed on a thermal time scale, LFVP varied between 158 and 354 degree-days above a base temperature of $0.5^{\circ} \mathrm{C}$.

Across all batches, the mean LFVP was similar for the two cultivars, with values of 20.1 and 21.3 days for AGS 2000 and USG 3209, respectively. However, LFVP was longer for USG 3209 than for AGS 2000 in six of the batches, and shorter for USG 3209 than for AGS 2000 in only one batch. The greatest difference in LFVP between the two cultivars in a single batch was 5 days. Linear regression of the rate of lesion development during the latent period (1/LFVP) on mean temperature after inoculation provided a similar fit for both AGS 2000 and USG 3209 (data not shown). A test of homogeneity of slopes did not reveal any significant $(P=0.52)$ difference between regression lines for AGS 2000 and USG 3209, and when the data for the two cultivars were combined, the relationship between 1/LFVP and mean temperature was best described $\left(r^{2}=0.81, P<0.0001\right)$ by a linear equation (Fig. 2$)$. The reciprocal of the slope in the linear regression equation, $y=0.0072$ $+0.0026 x$, i.e., $1 / 0.0026$ (=384.6 degree-days) represents the ther-



Fig. 2. Inverse of latent period (1/days) from inoculation of Stagonospora nodorum to observation of the first lesions with pycnidia (LFVP) for all batches of winter wheat cultivars AGS 2000 and USG 3209. The line depicts a fit of batch mean data to a linear model based on linear regression analysis. AGS 2000 is highly susceptible and USG 3209 is moderately susceptible to Stagonospora nodorum blotch. Mean temperature is the average of maximum and minimum temperatures to which each batch was exposed under outdoor conditions. 
mal time required above a base temperature of $0^{\circ} \mathrm{C}$ for completion of the latent period.

Increase in lesions with pycnidia. The increase in the number of lesions with pycnidia during the experimental period varied widely among seedling batches following inoculation with $S$. nodorum. For example, the time from inoculation to maximum number of observed lesions with pycnidia (L100) differed among batches and ranged from 25 to 54 days. Similarly, there were substantial differences among seedling batches in symptom duration ranging from 10 to 30 days across all batches. These differences in both $L 100$ and symptom duration were related to differences in temperature, with the shortest times for both variables being recorded for seedling batches exposed to the warmest temperatures. As with $1 / \mathrm{LFVP}$, regression analysis for the relationship between $1 / L 100$ and mean exposure temperature of batches for combined
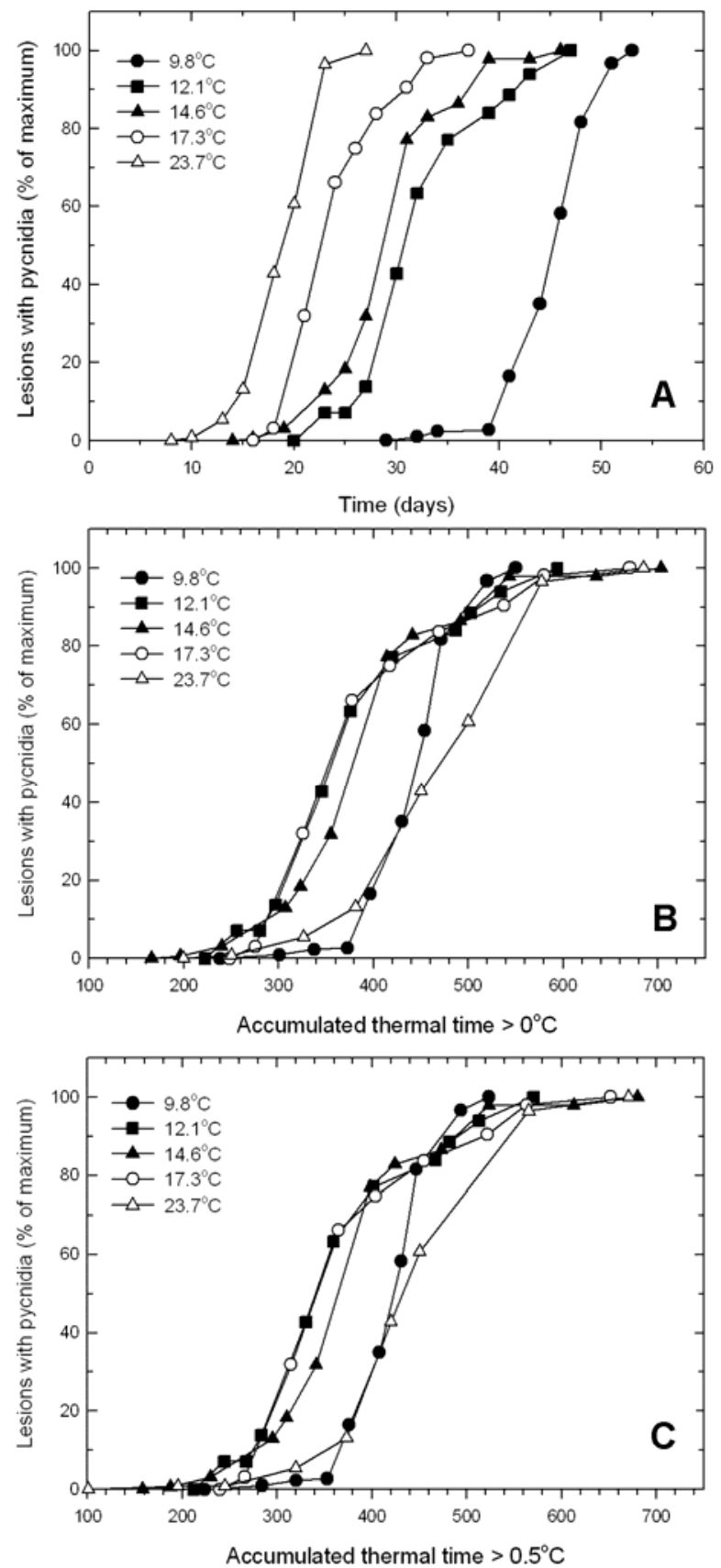

Fig. 3. Temporal increase in number of lesions with pycnidia (as percentage of maximum number of observed lesions with pycnidia) for five batches of wheat cultivar AGS 2000. Data are presented on three different time scales: A, days of exposure; $\mathbf{B}$, accumulated thermal time above base temperature of $0^{\circ} \mathrm{C}$; and $\mathbf{C}$, accumulated thermal time above a base temperature of $0.5^{\circ} \mathrm{C}$. data across AGS 2000 and USG 3209 was best described $\left(r^{2}=\right.$ $0.87, P<0.0001$ ) by a linear equation (data not shown).

For a set of batches of AGS 2000 (Fig. 3) and USG 3209 (Fig. 4) selected to depict the range of mean temperatures during the study, there were distinct differences among batches in the development of lesions with pycnidia on both physical- and thermal-time scales. For both cultivars, differences among batches in LFVP, L100, and symptom duration were reduced by using a thermal-time scale (degree-days above a base temperature of $0^{\circ} \mathrm{C}$, Figs. 3B and 4B) instead of the physical-time scale (Figs. 3A and 4A). For a base temperature of $0^{\circ} \mathrm{C}$, the observed mean values for the symptom lag were 238 and 276 degree-days for AGS 2000 and USG 3209, respectively, while observed mean values for symptom lag were 244 and 289 degree-days above a base temperature of $0.5^{\circ} \mathrm{C}$ for AGS 2000 and USG 3209, respectively.
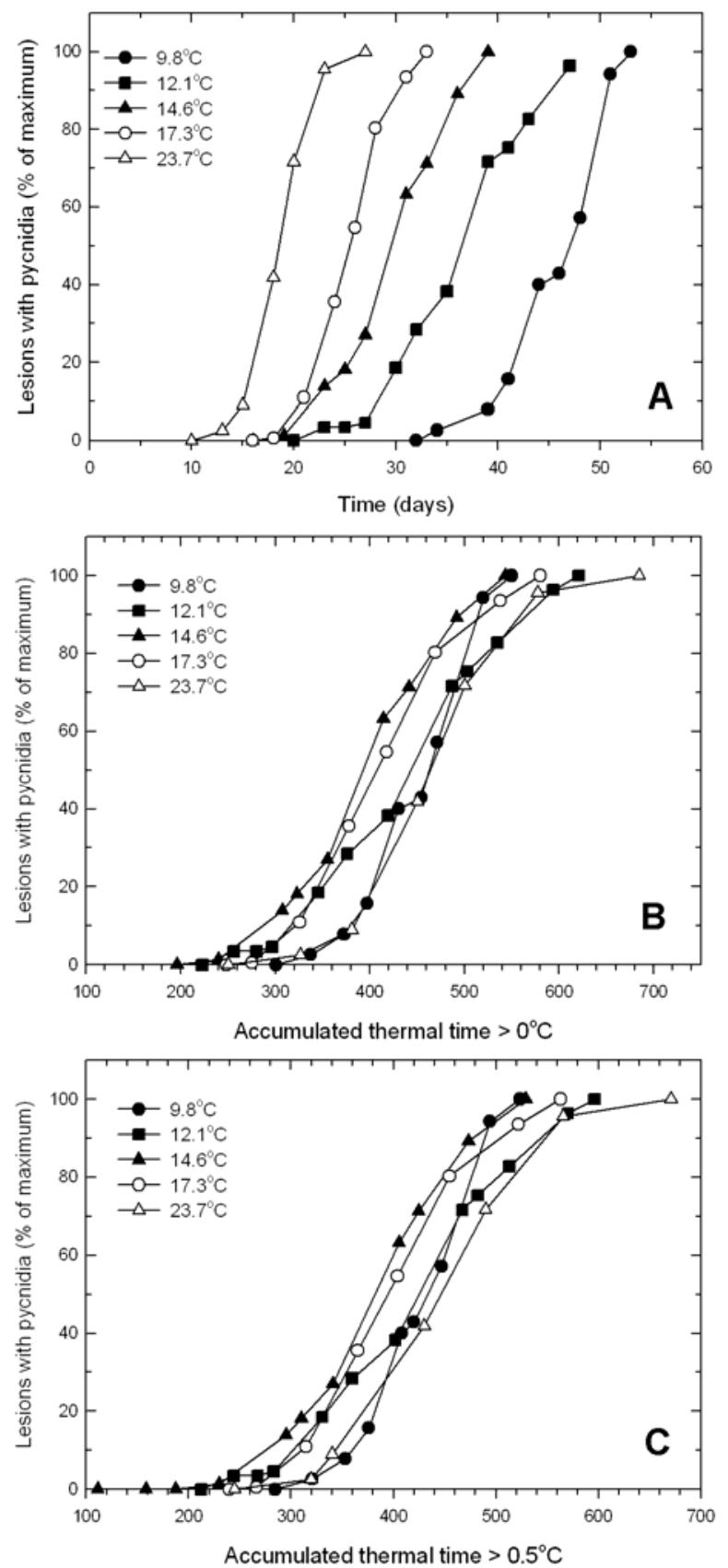

Fig. 4. Temporal increase in number of lesions with pycnidia (as percentage of maximum number of observed lesions with pycnidia) for five batches of winter wheat cultivar USG 3209. Data are presented based on three different time scales: A, days of exposure; $\mathbf{B}$, accumulated thermal time above $0^{\circ} \mathrm{C}$; and $\mathbf{C}$, accumulated thermal time above a base temperature of $0.5^{\circ} \mathrm{C}$. 
Model for development of lesions with pycnidia. The shifted cumulative gamma distribution model for describing the increase in number of lesions with pycnidia over accumulated thermal time resulted in smaller error mean squares (less variation) when thermal time was calculated based on a base temperature of $0.5^{\circ} \mathrm{C}$ than on a base temperature of $0^{\circ} \mathrm{C}$, for both AGS 2000 (Fig. 3C) and USG 3209 (Fig. 4C). For example, the error mean squares were about $6 \%$ lower for a base temperature of $0.5^{\circ} \mathrm{C}$ than for a base temperature of $0^{\circ} \mathrm{C}$. Use of base temperatures $>0.5^{\circ} \mathrm{C}$ or base temperatures $<0^{\circ} \mathrm{C}$ to optimize model parameters resulted in lack of convergence of the model. Thus, final parameter estimates for the model used in subsequent analysis were based on thermal time calculated using a base temperature of $0.5^{\circ} \mathrm{C}$.

The final gamma distribution model provided a good fit $(P<$ $0.0001)$ to the increase in number of lesions with pycnidia with accumulated thermal time for both AGS 2000 and USG 3209. Parameter estimates for both cultivars were significantly $(P<0.05)$ different from zero for combined data across all batches (Table 1). The correlation coefficients between observed and predicted number of lesions with pycnidia were 0.927 and 0.939 for AGS 2000 and USG 3209, respectively. Plots of residuals versus predicted number of lesions with pycnidia did not show a systematic pattern in the residuals for both cultivars (data not shown).

The estimated values for the symptom lag (parameter $p$ in the model) were 249.4 and 255.6 for AGS 2000 and USG 3209, respectively (Table 1 ), and these values were similar to the symptom lag values obtained from observed data, especially for AGS 2000 using a base temperature of $0.5^{\circ} \mathrm{C}$. Estimates of accumulated time from inoculation to $L 5$ and $L 50$ were slightly higher for AGS 2000 than for USG 3209 (Table 2). However, the difference in the range of the latent period was comparable for the two cultivars (Table 2$)$. No heterogeneity $(P=0.48)$ was observed in the slopes of $1 / L 50$ against mean batch temperatures when graphs for AGS 2000 and USG 3209 were plotted separately. A plot of the inverse of $L 50$ against mean temperature was also best described by a linear equation $\left(r^{2}=0.93, P<0.0001\right)$ for combined data across the two cultivars (Fig. 5).

\section{Discussion}

The effect of temperature on the latent period of SNB was investigated using two winter wheat varieties that differed in their reaction to $S$. nodorum. In both cultivars, the daily rate of development of lesions with pycnidia was linearly related to the mean temperature during the latent period. The thermal time required for completion of the latent period of SNB was estimated as 384.6 degree-days. When examined on a physical-time scale, the increase in lesions with pycnidia with time varied considerably between batches and was dependent on the time of year. However, the increase in number of lesions with pycnidia was observed to be similar at different times of year when plotted on a thermal-time scale. Further, the increase in lesions with pycnidia on a thermal-time scale was well described using a shifted gamma distribution model.

Table 1. Parameters and statistics for the cumulative gamma distribution model used to describe the increase in the number of lesions with pycnidia with accumulated thermal time following inoculation of winter wheat cultivars AGS 2000 and USG 3209 with Stagonospora nodorum

\begin{tabular}{lccccc}
\hline & \multicolumn{2}{c}{ AGS 2000 $^{\mathbf{a}}$} & & \multicolumn{2}{c}{ USG 3209a $^{\mathbf{n}}$} \\
\cline { 2 - 3 } \cline { 5 - 6 } Parameter $^{\mathbf{b}}$ & Estimate & S.E. & & Estimate & S.E. \\
\hline$\alpha$ & 1.79 & 0.66 & & 2.06 & 0.84 \\
$\beta$ & 85.01 & 0.00 & & 73.85 & 0.00 \\
$p$ & 249.40 & 42.71 & & 255.60 & 54.13 \\
Error mean squares $\left(\mathrm{s}^{2}\right)$ & 4.93 & $\ldots$ & & 3.03 & $\ldots$ \\
\hline
\end{tabular}

a AGS 2000 and USG 3209 are susceptible and moderately susceptible to Stagonospora nodorum blotch, respectively. S.E. is the standard error for the estimate of the model parameter.

${ }^{\mathrm{b}}$ Parameter estimates for $\alpha$ (shape parameter), $\beta$ (scale parameter), and $p$ (lag parameter) are given in degree-days based on a base temperature of $0.5^{\circ} \mathrm{C}$. For AGS 2000, $\mathrm{df}=172$, and for USG 3209, $\mathrm{df}=169$.
Most of the previous studies $(19,31,34)$ on the latent period of $S$. nodorum were conducted under controlled conditions where temperature was held constant, and only a few studies $(20,32)$ have investigated the latent period of $S$. nodorum under field conditions. In the latter studies, the latent period was reported to be in the range of 7 to 34 days, which, depending on the mean ambient temperature, corresponds to a thermal-time accumulation of 250 to 500 degree-days above a base temperature of $0^{\circ} \mathrm{C}$. The accuracy in estimating thermal time to first sporulating lesions with increasing temperature can be lost by using long intervals and/or fixed intervals for assessing lesions with pycnidia (21). In previous studies $(15,19,31)$ as well as in the present study, observations of lesions with pycnidia were made every 1 to 3 days. However, even when the interval of observations is relatively short, defining latent period using an inverse transformation (1/days) results in errors that are greater at higher temperatures or shorter latent periods. Analysis of the relationship between the increasing number of lesions with pycnidia and accumulated thermal time using the cumulative gamma distribution model results in reduced errors due to either less frequent assessments or irregular thermal-time periods between assessments (28). The relatively short interval for assessing lesions with pycnidia combined with the modeling approach used in this study maximized the accuracy of thermal-time period

Table 2. Estimates of thermal time required to fulfill the latent period of Stagonospora nodorum inoculated on seedlings of blotch, defined as either time from inoculation to $5 \%$ of maximum lesions with pycnidia $(L 5)$, or $50 \%$ of maximum lesions with pycnidia ( $L 50)$, and range in length of latent period for winter wheat cultivars AGS 2000 and USG 3209

\begin{tabular}{lccc}
\hline Defined latent period & Cultivar $^{\mathbf{a}}$ & $\begin{array}{c}\text { Thermal time } \\
\text { (degree-days) }^{\mathbf{b}}\end{array}$ & $\begin{array}{c}\text { Standard } \\
\text { error }\end{array}$ \\
\hline 5\% of maximum lesions & AGS 2000 & 310.5 & 4.12 \\
(L5) & USG 3209 & 307.1 & 3.61 \\
50\% of maximum lesions & AGS 2000 & 335.7 & 6.97 \\
(L50) & USG 3209 & 325.9 & 5.64 \\
Range in length of latent & AGS 2000 & 25.2 & $\ldots$ \\
period $(L 50-L 5)$ & USG 3209 & 18.8 & $\ldots$ \\
\hline
\end{tabular}

a AGS 2000 is susceptible, while USG 3209 is moderately susceptible to Stagonospora nodorum blotch.

b Values are mean estimates of the defined latent period generated by modeling increase in number of lesions with pycnidia with thermal time. Thermal time is in accumulated degree-days above a base temperature of $0.5^{\circ} \mathrm{C}$, estimated from the cumulative gamma distribution model of number of lesions with pycnidia.

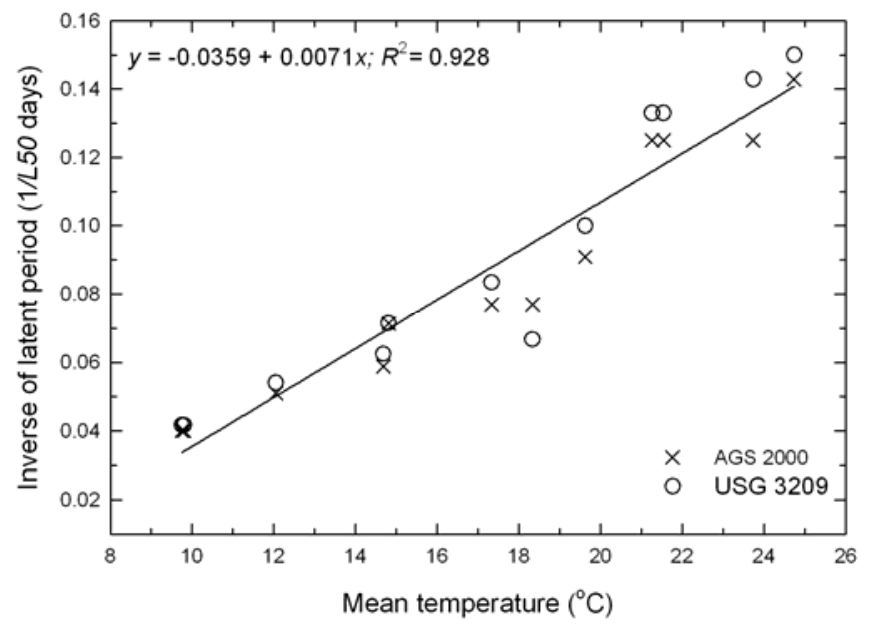

Fig. 5. Inverse of latent period (1/days) from inoculation of Stagonospora nodorum to $50 \%$ of the maximum number of lesions with pycnidia $(L 50)$ for all batches of winter wheat cultivars AGS 2000 and USG 3209. The line depicts a fit of batch mean data to a linear model based on linear regression analysis. AGS 2000 is highly susceptible and USG 3209 is moderately susceptible to Stagonospora nodorum blotch. Mean temperature is the average of maximum and minimum temperatures to which each batch was exposed under outdoor conditions. 
estimates. The model estimated thermal time from inoculation to the appearance of the first lesion with pycnidia to approximately 253 degree-days, which is on the lower end of the 250 to 500 degree-days range reported in the previous studies $(15,19,31)$. Thus, it is likely that previous field studies on the latent period of SNB $(15,19,31,32)$ may have overestimated the latent period of $S$. nodorum.

In this study, wheat plants were exposed to a range of temperature that represents typical variation in temperatures during the winter wheat growing season in the southeastern United States. Although $S$. nodorum isolates and wheat genotypes used in previous studies $(8,31,34)$ on the latent period of $S$. nodorum are different from those used in the present study, a general comparison of the magnitude of the latent periods is still possible. Our observations of the latent period of $S$. nodorum with regard to the first appearance of lesions with pycnidia are broadly similar to those previously reported in the United States $(8,34)$ in a controlled environment with a temperature range of 18 to $25^{\circ} \mathrm{C}$. Our results are also similar to those reported in Europe in controlledenvironment experiments for a temperature range of 10 to $20^{\circ} \mathrm{C}$ (31). However, our estimates of the time to first lesions with visible pycnidia are substantially lower than those reported in previous studies (31) at lower temperatures. For example, in the present study, the time to first lesions with pycnidia was 35 days at lowest temperatures $\left(<5^{\circ} \mathrm{C}\right)$, while 49 days was the corresponding time to first lesions with pycnidia in the study by Shearer and Zadoks (31). Such differences may be attributed to other factors such as age of plants at inoculation and the temperatures when plants were inoculated. For example, in the study by Shearer and Zadoks (31), plants were inoculated at the three-leaf stage at a temperature of $17^{\circ} \mathrm{C}$, while in the present study, wheat plants were inoculated at the four-leaf stage at a temperature of $21^{\circ} \mathrm{C}$. In this study, the standardization of the inoculation procedure across all seedling batches eliminated the environment as a source of variation during inoculation and incubation. This allowed our observations to reflect only the postinfection effects of temperature on the latent period of $S$. nodorum under outdoor conditions.

This is the first study that documents a linear relationship between the inverse of the latent period and mean temperature for $S$. nodorum on wheat. Similar observations of a linear relationship between mean temperature and inverse of latent period have been reported for Mycosphaerella capsellae on oilseed rape (14), $M$. graminicola infecting winter wheat (21), and $P$. hordei on spring barley (4). To link crop growth to $P$. hordei development on barley, Beresford and Royle (4) reported a pathochron of 1.95, indicating that 1.95 phyllochrons (emergence of successive leaf layers) elapsed during one $P$. hordei latent period. This suggests that the barley crop is likely to grow away from sources of inoculum in the lower canopy, thereby reducing the probability of spore arrival on the upper leaves. This has also been demonstrated for M. graminicola in winter wheat (22). In order to describe the latent period of $S$. nodorum in terms of the rate at which wheat leaves emerge, it is necessary to also describe the leaf emergence in terms of thermal time. In wheat, leaf emergence rate is constant throughout the development of the crop, and a leaf emerges after each 110 degreedays (17). In this study, the rate of development during a given latent period of $S$. nodorum was estimated as 384.6 degree-days. Applying the pathochron concept, 3.5 phyllochrons $(=384.6 / 110)$ will, therefore, elapse during the latent period of $S$. nodorum. Thus, the winter wheat crop is likely to grow away from sources of $S$. nodorum inoculum, reducing the probability of pycnidiospore arrival on the successive upper leaf layers.

The findings reported here have direct utility in the management of SNB. For wheat diseases such as Septoria leaf blotch and SNB, low levels of disease are not likely to cause significant yield losses (36). Thus, disease thresholds defined as either $L 5$ or $L 50$ are likely to be more relevant in guiding profitable fungicide applications. The model developed in this study can also be used to estimate a wide range of potentially useful threshold values between $L 5$ and L50. Decisions in the timing of fungicide applications are based on thresholds for $S$. nodorum that are determined when indicator leaves are sampled for lesions with pycnidia $(35,36)$. Our results show that the rate of leaf emergence relative to the length of the latent period of $S$. nodorum is high, and therefore, host growth is likely to affect expression of the disease. This finding has implications in monitoring the disease during the season. For example, to eliminate bias due to crop growth, it should be recognized that at any given time, there will be leaves in the crop that will not contribute to disease and should be ignored. Thus, instead of averaging disease over leaves of different ages at each assessment date, disease should be monitored on one or more age classes of leaves that are more than one latent period old. In addition, sampling and disease assessment should be adjusted for thermal time, and shorter intervals should be used as the temperature increases.

Host disease resistance is generally expressed as reduced rates of disease increase, for example, longer latent periods in the case of polycyclic diseases (16). In this study, latent period variables, LFVP, L5, and L50, and model parameters did not differ substantially between cultivars AGS 2000 and USG 3209, although these two cultivars differ in their overall resistance to SNB. A similar observation was reported for $M$. graminicola on winter wheat, where negligible differences in latent period of Septoria tritici blotch were observed between a moderately resistant and a highly susceptible cultivar (21). One possible explanation for the lack of cultivar differences with respect to the latent period is that other disease resistance components, such as lesion size, rate of sporulation, or even toxin sensitivity, are probably more important than the latent period in determining overall resistance in the S. nodorumwheat pathosystem. Additional studies to determine how these other components of disease resistance relate to the latent period would be useful in providing guidance for the control of Stagonospora nodorum blotch.

\section{Literature Cited}

1. Abramowitz, M., and Stegun, I. A. 1964. Handbook of Mathematical Functions with Formulas, Graphs and Mathematical Tables. National Bureau of Standards Applied Mathematics Series 55. National Bureau of Standards, Washington, DC.

2. Bathgate, J. A. L. 2001. Ascospores are the sources of inoculum of Phaeosphaeria nodorum, P. avenaria f. sp. avenaria and Mycosphaerella graminicola in Western Australia. Aust. Plant Pathol. 30:317-322.

3. Bennett, R. S., Milgroom, M. G., Sainudiin, R., Cunfer, B. M., and Bergstrom, G. C. 2007. Relative contribution of seed-transmitted inoculum to foliar populations of Phaeosphaeria nodorum. Phytopathology 97:584591.

4. Beresford, R. M., and Royle, D. J. 1988. Relationships between leaf emergence and latent period for leaf rust (Puccinia hordei) on spring barley, and their significance for disease monitoring. J. Plant Dis. Prot. 95:361-371.

5. Bhathal, J. S., Loughman, R., and Speijers, J. 2003. Yield reduction in wheat in relation to leaf disease from yellow (tan) spot and Septoria nodorum blotch. Eur. J. Plant Pathol. 109:435-443.

6. Bockus, W. W., and Claassen, M. M. 1992. Effects of crop rotation and residue management practices on severity of tan spot winter wheat. Plant Dis. 76:633-636.

7. Bowman, D. 2007. North Carolina Measured Crop Performance: Small Grains 2007. Report No. 220. North Carolina State University, Raleigh, NC.

8. Cunfer, B. M., Stooksbury, D. E., and Johnson, J. W. 1988. Components of partial resistance to Leptosphaeria nodorum among seven soft red winter wheats. Euphytica 37:129-140.

9. Eyal, Z. 1999. The septoria tritici and stagonospora nodorum blotch diseases of wheat. Eur. J. Plant Pathol. 105:629-641.

10. Eyal, Z., Sharen, A. L., Prescott, J. M., and van Ginkel, M. 1987. The Septoria diseases of wheat: Concepts and methods of disease management. International Maize and Wheat Improvement Center (CIMMYT), Mexico, D.F., Mexico.

11. Gilbert, J., and Tekauz, A. 1993. Reaction of Canadian spring wheats to Septoria nodorum and the relationship between disease severity and yield components. Plant Dis. 77:398-402.

12. Holloway, D. L. 1979. Evidence that ethirimol may interfere with adenine metabolism during primary infection of barley powdery mildew. Pestic. Biochem. Physiol. 10:181-189.

13. Holmes, S. J. I., and Colhoun, J. 1974. Infection of wheat by Septoria nodorum and $S$. tritici in relation to plant age, air temperature and relative humidity. Trans. Br. Mycol. Soc. 68:329-338.

14. Inman, A. J., Fitt, B. D. L., Welham, S. J., Evans, R. L., and Murray, D. A. 
1997. Effects of temperature, cultivar and isolate on the incubation period of white leaf spot (Mycosphaerella capsellae) on oilseed rape (Brassica napus). Ann. Appl. Biol. 130:239-253.

15. Jeger, M. J., Jones, D. G., and Griffiths, E. 1983. Components of partial resistance of wheat seedlings to Septoria nodorum. Euphytica 32:575-584.

16. Johnson, C. S., Beute, M. K., and Ricker, M. D. 1986. Relationship between components of resistance and disease progress of early leaf spot on Virginia-type peanut. Phytopathology 76:495-499.

17. Kirby, E. J. M. 1994. Identification and Prediction of Growth Stages of Wheat Development for Management Decisions. Project Report No. 90. Home-Grown Cereals Authority, London, UK.

18. Krupinsky, J. M. 1999. Influence of cultural practices on Septoria/Stagonospora diseases. Pages 105-110 in: Septoria and Stagonospora Diseases of Cereals: A Compilation of Global Research. International Maize and Wheat Improvement Center (CIMMYT), Mexico, D.F., Mexico. Published online.

19. Lancashire, P. D., and Jones, D. G. 1985. Components of partial resistance to Septoria nodorum in winter wheat. Ann. Appl. Biol. 106:514-553.

20. Loughman, R., Wilson, R. E., and Thomas, G. J. 1996. Components of resistance to Mycosphaerella graminicola and Phaeosphaeria nodorum in spring wheats. Euphytica 89:377-385.

21. Lovell, D. J., Hunter, T., Powers, S. J., Parker, S. R., and Van den Bosch, F. 2004. Effect of temperature on latent period of Septoria leaf blotch on winter wheat under outdoor conditions. Plant Pathol. 53:170-181.

22. Lovell, D. J., Parker, S. R., Hunter, T., Royle, D. J., and Coker, R. R. 1997. Influence of crop growth and canopy structure on the risk of epidemics by Mycosphaerella graminicola (Septoria tritici) in winter wheat. Plant Pathol. 46:126-138.

23. Lovell, D. J., Powers, S. J., Welham, S. J., and Parker, S. R. 2004. A perspective on the measurement of time in plant disease epidemiology. Plant Pathol. 53:705-712.

24. McKendry, A. L., Henke, G. E., and Finney, P. L. 1995. Effects of Septoria leaf blotch on soft red winter wheat milling and baking quality. Cereal Chem. 72:142-146.

25. Milus, E. A. 1994. Effects of leaf rust and Septoria leaf blotch on yield and test weight of wheat in Arkansas. Plant Dis. 78:55-59.
26. Nelson, L. R., Morey, D. D., and Brown, A. R. 1974. Wheat cultivar responses to severe glume blotch in Georgia. Plant Dis. Rep. 58:21-23.

27. Paveley, N. D. 1999. Integrating septoria risk variables. Pages 230-250 in: Septoria on Cereals: A Study of Pathosystems. J. A. Lucas, P. Bowyer, and H. M. Anderson, eds. CABI Publishing, Wallingford, UK.

28. Powers, S. J., Brain, P., and Barlow, P. W. 2003. First-order differential equation models with estimable parameters as functions of environmental variables and their application to a study of vascular development in young hybrid aspen stems. J. Theor. Biol. 222:219-232.

29. Shah, D., Bergstrom, G. C., and Ueng, P. P. 1995. Initiation of Septoria nodorum blotch epidemics in winter wheat by seedborne Stagonospora nodorum. Phytopathology 85:452-457.

30. Shaw, M. W. 1990. Effects of temperature, leaf wetness and cultivar on the latent period of Mycosphaerella graminicola on winter wheat. Plant Pathol. 39:255-268.

31. Shearer, B. L., and Zadoks, J. C. 1972. The latent period of Septoria nodorum in wheat. 1 . The effect of temperature and moisture treatments under controlled conditions. Neth. J. Plant Pathol. 78:231-241.

32. Shearer, B. L., and Zadoks, J. C. 1974. The latent period of Septoria nodorum in wheat. 2 . The effect of temperature and moisture treatments under field conditions. Neth. J. Plant Pathol. 80:48-60.

33. Sommerhalder, R. J., McDonald, B. A., and Zhan, J. 2006. The frequencies and spatial distribution of mating types in Stagonospora nodorum are consistent with recurring sexual reproduction. Phytopathology 96:234-239.

34. Stooksbury, D. E., Johnson, J. W., and Cunfer, B. M. 1987. Incubation period and latent period of wheat for resistance to Leptosphaeria nodorum. Plant Dis. 71:1109-1112.

35. Stromberg, E. L. 2010. Small Grains. In: Pest Management Guide: Field Crops 2010. Virginia Cooperative Extension Publ. 456-016. Virginia Polytechnic Institute and State University, Blacksburg, VA.

36. Verreet, J. A., and Hoffmann, G. M. 1990. A biologically oriented threshold decision model for control of epidemics of Septoria nodorum in wheat. Plant Dis. 74:731-738.

37. Verreet, J. A., Klink, H., and Hoffmann, G. M. 2000. Regional monitoring for disease prediction and optimization of plant protection measures: The IPM wheat model. Plant Dis. 84:816-826. 